\title{
La historia de la enseñanza de la historia: un saber escolar inestable
}

The history of the teaching of history: a unestable school knowledge

A história do ensino da história: um saber escolar instável

El presente artículo es resultado del proyecto de investigación que condujo a la tesis de Maestría en Historia en la Pontificia Universidad Javeriana: La configuración del saber pedagógico para la enseñanza de la historia.

Mireya González Lara

Licenciada en Ciencias Sociales, Especialista en Enseñanza de la Historia y Magister en Historia. Actualmente coordina el Instituto para el desarrollo y la Innovación Educativa (IDIE) de la Organización de Estados Iberoamericanos (OEI) y es docente catedrática del Departamento de Historia de la Pontificia Universidad Javeriana.

miregonzalezlara@yahoo.es 


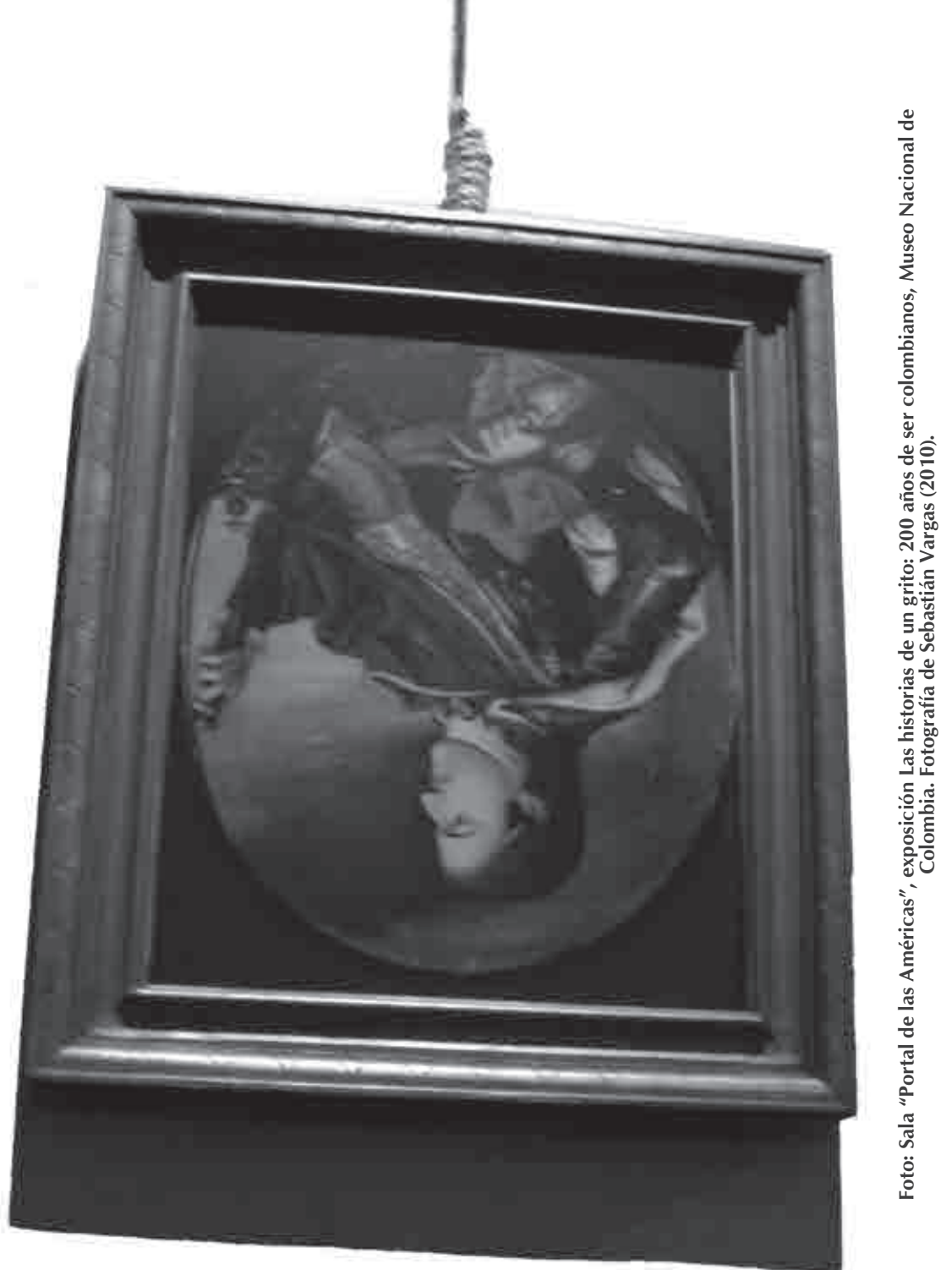

En 1819 los vecinos de la Villa de Honda solicitaron colgar públicamente, durante lueve horas, los retratos de los reyes ue se encontraban en la secretaría de la udad, para lueqo quemarlos a la vista 


\section{Resumen}

La historia de la enseñanza de la historia en Colombia, tradicionalmente ha estado articulada a la trayectoria que ha marcado la formalización de la historia como disciplina académica universitaria, configurando su enseñanza como un ejercicio dependiente, subordinado y siempre deficitario de los avances logrados desde su investigación. La reciente emergencia del campo investigativo de la historia de los saberes escolares en Colombia, se ha venido constituyendo en una posibilidad de indagar por la historia de la enseñanza de la historia, desde otros referentes teóricos y metodológicos, que además, de potenciar la categoría de "saber pedagógico", permite la exploración de otros discursos que han configurado históricamente este saber, asociados a la formación de docentes, a las políticas públicas, a los textos escolares y a la investigación sobre este campo. El presente artículo, aprovechando la coyuntura de los Bicentenarios, intenta mostrar esta perspectiva teórica y algunos esbozos iniciales sobre las formas que ha tomado la enseñanza de la historia a lo largo del siglo XX y la primera década del siglo XXI.

\section{Palabras clave}

Enseñanza de la historia, historia escolar, historia académica, saber pedagógico.

The history of teaching of history in Colombia has traditionally been articulated to the path that has marked the formalization of history as an academic university field, setting his teaching as a dependent exercise, subordinated and in always in debt with the progress from research. The relatively recent emergence of the field of research in the history of school knowledge in Colombia, has been constituted in a chance to inquire about the history of the teaching of history from other theoretical and methodological references, in addition to enhance the category of "pedagogical knowledge ", and allows the exploration of other discourses that have historically shaped this knowledge, associated with teacher training, public policy, school textbooks and research on this field. This article, drawing on the situation of the Bicentennial, attempts to show this theoretical perspective and some initial sketches of the forms it has taken the teaching of history throughout the twentieth century and the first decade of the century.

Key words

Teaching of history, school history, academic history, pedagogical knowlegde 
A história do ensino da história em Colômbia tradicionalmente foi articulada com a trajetória que marcou a formação da história como disciplina acadêmica universitária, o que configurou seu ensino como um exercício dependente, subordinado e sempre deficitário dos avances alcançados com sua investigação. A relativamente nova emergência do campo investigativo da história dos saberes escolares em Colômbia se constituiu como uma possibilidade para a indagação da história do ensino da história, desde outros referentes teóricos e metodológicos, que, além de potenciar a categoria de "conhecimento pedagógico”, permite a exploração de outros discursos que configuraram historicamente este saber, em sociedade com a formação de professores, as políticas públicas, os textos escolares e a investigação. O presente artigo, no contexto da conjuntura dos dois bicentenários, tenta mostrar esta perspectiva teórica e alguns esboços das formas que teve o ensino da história ao longo do século 20 y na primeira década do século 21.

Palavras-chave

Ensino da história, história escolar, história acadêmica, saber pedagógico.

El año 2010 fue la apertura a un ciclo importante de conmemoraciones para los países latinoamericanos, se cumplieron doscientos años del inicio de sus procesos de independencias de la Monarquía Española, y a la vez de los primeros esbozos y experimentos políticos como estados-naciones autónomas. Hace doscientos años, comenzó a tomar forma lo que hoy conocemos como Colombia; la conformación de su territorio, de su población, de las formas de organización, sus instituciones, leyes y en especial, la emergencia de esa "voz interna" que nombra a cada sujeto de manera específica: colombiano y colombiana.

Hablar del Bicentenario, entonces, es hablar de los orígenes de una idea, de un sentimiento y de un referente; de los mitos fundacionales de lo que se ha dado en llamar Colombia. Es una efeméride que compromete el sentido de lo nacional, en su construcción a lo largo de dos siglos, cambiante o estática, pero siempre resignificada en su contenido y expresión. El Bicentenario es una acontecimiento que indaga por la manera como se han configurado los Estados, y en especial, las naciones, esas “comunidades imaginadas", que han servido de referente de identidad para algunos sujetos, ciudadanos, hombres y mujeres que habitan estos territorios. 
Las conmemoraciones para los doscientos años de la independencia, han permitido constatar el lugar de poder que representa el ámbito de la memoria política; ¿De cuál independencia estamos hablando? Se pregunta Elizabeth Castillo (2010: 6) al interpelar críticamente los efectos simbólicos y estéticos que se crearon durante el 2010, en torno a un "hito" fundante de la nacionalidad, cuya fragilidad hoy más que nunca se hace evidente.

Muchas son las preguntas y las miradas que emergen a propósito de esta conmemoración; hacen parte de las tensiones y paradojas que atraviesa la experiencia nacional, incompleta e insuficiente. "Tensiones que provienen de una larga historia de exclusiones e invisibilidades que no han permitido alterar las voces y las figuras de nuestra memoria oficial" puntualiza Castillo (2010: 6).

Indagar sobre los mecanismos y dispositivos que han permitido o no, la emergencia de ese sentimiento y emoción, de esa "voz interna" en cada uno de los sujetos, seguramente, sus posibles respuestas conducen e indagan por la escuela, por los sujetos de la enseñanza, los maestros, y por el saber escolar al que se le ha encargado tal función, como es la historia y/o las ciencias sociales. Entonces, las preguntas continúan, ¿cómo actúa este dispositivo escenarios-sujetos y saberes en la emergencia de este referente identitario? ¿Sigue siendo efectivo este dispositivo en la actualidad? ¿Qué tipo de referentes se construyen hoy en la Escuela? ¿Cuál es el lugar de las ciencias sociales en ella? ¿Qué debe saber un maestro de historia hoy?

\section{La configuración de un saber curricular: la enseñanza de la historia}

Nuestro punto de partida para organizar, priorizar, definir e intentar responder algunas de estas preguntas, ha sido considerar los saberes como construcciones históricas y también así el saber pedagógico; lo que conocemos hoy como la historia que se enseña ha tomado forma a lo largo del siglo XX y aún continúa en ese proceso en esta primera década del siglo XXI, no es un proceso terminado, decantado. La manera como se ha esculpido este saber escolar y su presencia hoy en la escuela, es un resultado que revela las acciones de poder, que durante más de una centuria han atravesado la constitución de las llamadas “ciencias” y su relación con la pedagogía.

En el ámbito de esta relación, se ha ido definiendo el lugar y el saber del maestro como el sujeto "[...] que soporta el método como distintivo de su oficio y su relación con el saber" y como "el que enseña por su relación con el saber y no por su relación con el método [...]” 
(Zapata, 2003: 181), siendo entonces la enseñanza y el método, los elementos que definen el núcleo del oficio del maestro. ¿Cuál es el lugar epistemológico del saber pedagógico del maestro? ¿Cómo se ha configurado ese saber pedagógico?

El Grupo de Historia de la Práctica Pedagógica en Colombia ha planteado, de diferentes formas, la condición dependiente, subalterna o de menor valía del saber pedagógico. Zuluaga advierte que "Entre los sujetos que de una u otra manera se relacionan con el discurso de las ciencias o de los conocimientos, hay uno de ellos cuya forma de relación designa una opresión cultural que se establece a través del método de enseñanza: ése es el maestro [...]" (1999: 156).

La razón fundamental que explica esta condición, obedece a la naturaleza epistemológica del saber pedagógico, pues, a diferencia de otros, define y fundamenta su objeto en su relación con otras disciplinas y en la práctica misma. Tempranamente, en el siglo XIX, ya se planteaba la naturaleza de este saber:

[...] el oficio del Maestro es enseñar [...] Visto está que la enseñanza es un arte que hay que estudiar. La ciencia que nos da a conocer este arte es la Pedagogía. Bien pudiera suceder que alguno aprendiera a enseñar asistiendo a buenas lecciones modelos e imitándolos en la práctica constante: pero en la época actual no satisface la mera rutina en la enseñanza, pues el maestro debe darse teóricamente cuenta de su modo de proceder en cualquier punto y caso en la enseñanza; debe saber dar razón por qué en cada uno de los casos ha obrado de esta y no de aquella manera. El maestro tiene, por lo tanto, que aprender la Teoría de la ciencia de enseñar. La práctica sin la teoría es mera rutina. La enseñanza pedagógica tiene que ser teórica y práctica [...] (Citado por Álvarez, 2008: 61).

La configuración del saber pedagógico se ha inscrito en los cánones modernos y positivistas de occidente; un saber que se diputa su lugar con las ciencias sociales, desde un paradigma que privilegia una comprensión social de la ciencia, en términos relacionados con la objetividad. La neutralidad, la dicotomía entre sujeto y objeto, cuya producción de conocimiento necesariamente se realiza a través del "método científico", y la naturaleza del conocimiento producido está asociada a la posibilidad de que sea verificable, generalizable, y la mayoría de las veces debe conducir a la generación de leyes. Un conocimiento que se produce y se juega su legitimidad, en lo que Lander nos propone como "las múltiples separaciones de occidente": cuerpo-mente, ciencia-sociedad, naturaleza-cultura, hombrenaturaleza (Lander, sf: 2-8). 
1. Ospina plantea que sobre la presencia del "positivismo" en la historiografía colombiana del siglo XIX, hay pocos estudios y el problema se agrava por la poca claridad sobre el tema. Por ejemplo, mientras el historiador Tovar Zambrano encuentra "un cierto sabor empirista positivista" en la obra de José Manuel Restrepo y en la de José Antonio Plazas, el historiador Jorge Orlando Melo piensa, contradiciendo a Zambrano, que no hubo "positivismo en sentido propio", y lo critica por confundir empirismo con positivismo. Para la exposición detallada de estos debates historiográficos, ver Ospina (2007). Por lo general, y en forma peyorativa, se entiende el "positivismo" como un "empirismo estrecho", un "humanismo erudito" o una "historiografía romántica". Otro gran problema, al abordar este estudio, es la dificultad que presenta la "sospechosa" relación entre la filosofía positivista y los pensadores cristianos, como ocurrió con la mayoría de los intelectuales colombianos del siglo XIX. Sin embargo, nos adelantamos a pensar, repitiendo las palabras de Leonardo Tovar González refiriéndose al mismo problema, pero a propósito de J.E. Caro, que "[...] no siempre las ideas religiosas riñeron con el progreso científico y técnico [...]" (2002: 35).
La doble condición del saber pedagógico, que referíamos anteriormente, leída en clave "científica”, posicionó a la pedagogía como una disciplina "carente" de un cuerpo de conceptos propios, "dependiente" de otras ciencias, "limitada" en sus decisiones epistemológicas y por tanto, deficitaria, en permanentemente aspiración de ser reconocida y legitimada como disciplina. Y a la vez, a los sujetos de la práctica pedagógica, definidos por este saber, se les ha venido "inventando" bajo la dominación cultural, de hombres y mujeres "[...] a quienes, por añadidura, se les ha exigido ser, ya implacables carceleros, ya paternales guías, ya minuciosos psicólogos u orientadores profesionales. Y desde ser agentes de bienestar social hasta sanadores de vicios físicos y heridas morales, desarmadores de pandillas juveniles y de bombas sociales, y algunas otras cosas [...]" (Saldarriaga, 2003: 16).

La paradoja sobre la que se constituye históricamente el saber pedagógico será también su "prisión”; aunque por su naturaleza quebranta los postulados básicos de la ciencia moderna, del "método científico" y del positivismo ${ }^{1}$, no podrá desmarcarse de ellos, en sus luchas por su legitimidad académica y social. En consecuencia, nos arriesgamos a afirmar que el saber pedagógico ha sido un saber doblemente violentado, recogiendo la categoría de Spivak; de un lado su naturaleza afincada en la práctica, la oralidad, la incertidumbre, propia de esa manera de ser y hacer similar al oficio del artesano, ha sido debilitada por esa búsqueda de un lugar en el soberbio territorio de las ciencias; pero por otro lado, en ese territorio ha sido denigrada, por cuanto se le ha dado un lugar de dependencia respecto a otras ciencias, restando importancia a las exploraciones propias que le permitan delinear, profundizar y legitimar sus especificidades como saber.

Esta ruta, ha permitido, que la "superioridad epistemológica" de las ciencias sociales, colonicen su territorio y por lo tanto, nombren a su manera lo que la pedagogía dejó de nombrar, los territorios de la práctica de los que dejó de ocuparse. Tras la promesa de la ciencia, la escuela, el maestro y la pedagogía se constituyeron como tales y de esta manera, siguiendo un fragmento de Lander (sf: 10), “[...] ocultar, negar, subordinar o extirpar toda experiencia o expresión cultural que no se ha correspondido con este deber ser que fundamenta a las ciencias sociales [...]".

Por supuesto, en este marco se ha establecido la relación de la historia y su enseñanza en la escuela ¿Por qué se enseña historia en la escuela? y ¿Qué historia se debe enseñar? Son preguntas que orientan las indagaciones, que nos dejan avanzar en nuestra comprensión sobre la manera cómo se han construido, los consensos 
y disensos sociales y políticos que han logrado en diferentes momentos, leer la potencia política y ética de un saber o de una disciplina. Saberes que logran un lugar en un plan de estudios o en un currículo escolar, y que en la mayoría de los casos no obedece a una racionalidad científica.

Cuando observamos estas indagaciones, constatamos que generalmente se ocupan - casi exclusivamente-de hacer explicitas las condiciones que permitieron la emergencia e institucionalización de la historia como disciplina científica, para luego ocuparse del devenir histórico de las "aplicaciones" o "adaptaciones" que se han logrado de ese conocimiento disciplinar para su enseñanza. Por esta razón, se ocupan de mirar por ejemplo; qué tanto la escuela de los Annales, los enfoques marxistas o los estudios culturales han permeado los contenidos y perspectivas de los planes de estudio o hasta que punto se hallan reflejados en los manuales escolares.

Este tipo de explicación sobre la enseñanza de la historia, muy fuerte por cierto en nuestro medio, se asienta en aquellas "verdades" instituidas, naturalizadas, familiarizadas, que refrendan las concepciones instrumentales de la pedagogía, donde el oficio del maestro se asocia sólo al método y no al saber. Desde esta concepción, en efecto, se subraya la condición marginal, dependiente y subordinada de la pedagogía en su relación con la historia, y se muestra entonces, que la historia que se enseña es definida en instancias académicas externas y extrañas a la escuela, en las que su preocupación fundamental es el rigor científico, es decir, cómo se ha producido ese conocimiento.

Los efectos de esta concepción en la pedagogía y en el maestro los vemos hoy, mientras que la primera debe garantizar la enseñanza de unas versiones resumidas, acortadas y en la mayoría de los casos, maltrechas de la historia que se investiga, los segundos deberán garantizar "el rigor científico" en la enseñanza de la historia y, para ello, el maestro deberá estar atento a la actualización permanente de los conocimientos sobre la historia que se produce por fuera de la escuela.

No obstante, durante las últimas décadas se han abierto nuevas perspectivas de estudios, acerca la relación pedagogía e historia, básicamente a partir del surgimiento de una tendencia en la historia de la educación, que indaga sobre la historia de los saberes escolares o curriculares, sospechando de la existencia de una relación de dependencia de la pedagogía con respecto a las ciencias, reconocida hoy en el campo educativo, sin problematización alguna. La convergencia de los estudios sobre la configuración del saber pedagógico y sobre las disciplinas científicas, para nuestro caso la historia, ha venido 
arrojando hallazgos que resultan por lo menos sorprendentes. Los trabajos genealógicos o en palabras de Cuesta, que trabajan la sociogénesis de las disciplinas escolares, han permitido delinear otro tipo de relaciones entre pedagogía e historia, que indica la estrecha relación entre ellas e incluso plantea la importancia de la pedagogía en la institucionalización de la disciplina histórica en Colombia.

Por su parte, Álvarez (2007) constata la presencia anticipada de la historia como saber escolar; la aparición de ésta es un acontecimiento asociado al surgimiento de los Estados nacionales; la nación fue lo que legitimó lo que debía ser enseñado o no, es decir que para el caso de la historia, su estatuto ideológico y moralizante fue lo que permitió su prematura presencia en los currículos escolares y aunque su correlato científico hacía presencia, inicialmente la fuerza legitimadora estaba en sus posibilidades axiológicas. Luego, ante el debilitamiento del nacionalismo, las dimensiones científicas de la historia son las que justifican su permanencia en el currículo escolar, sin el abandono total de su fuerza moralizante.

Cuesta ha insinuado para el caso español, que es "[...] la expansión de la literatura de divulgación histórica y de los libros de texto entre mediados y finales del siglo XVII lo que hace que la historia como saber empiece a dibujarse como una disciplina con algún futuro [...]" (1997: 67). De manera similar, Álvarez analiza el caso colombiano, planteando que en la institucionalización de la historia, la enseñanza y la pedagogía tuvieron un papel central, ya que desde allí se crea la necesidad de su formalización como disciplina científica; "[...] su institucionalización en la educación superior en parte se debe a la necesidad de ser enseñada en la escuela [...]" (2003: 286). Mientras que Betancourt advierte sobre la estrecha relación entre historia y pedagogía en la formación de historiadores, donde era impensable un docente que no hiciera investigación sobre lo que enseñaría (2007: 159), Lombana (2007) reconoce en la escuela Normal Superior del año 1936 un germinal intento, encaminado a la institucionalización de las ciencias sociales y de la historia como disciplina científica.

$\mathrm{Al}$ intentar quebrar la subordinación y dependencia del saber pedagógico con relación a la historia, emerge entonces cierta singularidad y autonomía del conocimiento histórico escolar y el conocimiento histórico científico, entendiendo que sus "lugares de producción" son completamente distintos. Por lo tanto, entenderíamos que no puede pretenderse que el conocimiento que circula en la escuela sea una adaptación o mera réplica del conocimiento científico, dado que la escuela y los maestros son escenarios y sujetos que también producen conocimiento, no solo lo reciclan. 
Cuando hablamos de "lugar de producción", nos estamos refiriendo a la categoría introducida por De Certeau (2006), que nos invita a poner en evidencia las condiciones que han permitido la emergencia de prácticas, discursos y saberes a través de los cuales toman forma determinadas maneras de ver, pensar y actuar de los sujetos. Entonces, las condiciones que han rodeado la aparición de la escuela, de la historia y del maestro son distintas de las que han permitido el surgimiento de las universidades y de sus docentes; estas condiciones se han transformado históricamente, y por lo tanto, sus intencionalidades y propósitos también.

Insistimos entonces, en que el conocimiento escolar y el disciplinar de la historia son diferentes, guardan unas especificidades justamente porque la intencionalidad, los métodos, los recursos, las instituciones y los sujetos que los producen son diferentes. Mientras que el primero se organiza, prioriza y dispone para unos propósitos que giran en torno a la enseñanza, en el segundo su organización la determina el rigor de su producción. Aunque aparentemente ambos conocimientos se rigen por el "método", la diferencia estaría en su finalidad: para uno el método garantiza su rigor y para el otro, la enseñanza. Cada una de las narrativas implicadas en estos dos tipos de conocimientos, tendrían entonces características diferentes, pues la del saber escolar estaría signada por el método, la relación con otras disciplinas y su resignificación desde la práctica, mientras que la narrativa de la historia científica estaría en el marco de este paradigma; planteamiento de un problema, referencias teóricas, trabajo sobre fuentes y producción de algunas explicaciones y comprensiones.

No obstante, la historia de esta relación entre historia y pedagogía se expresa hoy al preguntarnos sobre la enseñanza de la historia. Parte de lo que se ha venido comprendiendo es que el saber pedagógico para la enseñanza de la historia es un campo discursivo amplio y complejo, constituido por registros que proceden de diferentes escenarios, como los de decisión de política educativa los cuales prescriben el tipo de saber que deberá circular en la escuela, donde se intenta resolver el "para qué enseñar historia, lo que se debe enseñar y cómo se debe enseñar”. También de estos escenarios proceden registros dirigidos a definir el "quien debe enseñar y dónde se debe formar" ese sujeto para la enseñanza. Otro tipo de escenarios son los académicos, que tienen como propósito definir cuerpos conceptuales y metodológicos de las disciplinas específicas como la historia, pero también la pedagogía que en su relación con los escenarios de política o en desarrollo de su "función”, contribuyen a delinear "lo que se debe enseñar en las escuelas"; sus huellas se distinguen en la producción académica que han realizado los centros de investigación y las universidades sobre la enseñanza de la 
2. Esta primera ruta se caracteriza por los estudios y análisis de los documentos oficiales que pretenden

la configuración de la enseñanza de la historia: Marcos Generales, Lineamientos Curriculares, Estándares Básicos, entre otros. En esta perspectiva podemos ubicar los trabajos de CEPECS, y algunas tesis como la de Alexis Lombana.

3. Esta segunda ruta se caracteriza por los estudios e investigaciones sobre el campo de la enseñanza de la historia. Podemos referenciar los estados del arte de Cortés, González y Ospina de 2001 y el elaborado por Rodríguez y Acosta en el año 2007.

4. La tercera ruta propuesta ha sido promovida y acompañada por el Proyecto de Manuales Escolares -MANESque dirige la profesora Gabriel Ossenbach. Adicionalmente, la línea de trabajo sobre Manuales Escolares del Departamento de Historia de la Pontificia Universidad Javeriana ha aportado con los trabajos de Saldarriaga, Gualteros y Ospina. historia. En estos escenarios, generalmente configurados desde las políticas educativas, pero en algunos casos con rangos importantes de autonomía, que tienen como propósito la formación de los sujetos para la enseñanza, donde coinciden discursos de la política y de las disciplinas, organizados con fines formativos.

Estos grandes y complejos escenarios, en la medida en que contienen diferentes propósitos y finalidades, se han configurado históricamente de diferente manera y sus formas de relación también han cambiado. Hemos constatado que la complejidad que hoy manifestamos frente a "lo que debe saber un maestro para la enseñanza de la historia" y el proceso que él hace para tomar las decisiones en torno a ello, procede igualmente del abandono paulatino de la acción "reguladora" del Estado, sobre el manejo de lo social, lo que no significa menor control o vigilancia. Hasta la década de los cincuentas, con algunas variaciones, la enseñanza de la historia tomaba forma a través del control del Estado, incidiendo, controlando o regulando cada uno de los componentes que para el momento definían el núcleo de su acción; programas-textos-estatuto y formación docente. A partir de los años sesentas, ya no solamente se transforma el lugar y la función del Estado y su relación con la academia, sino además la concepción de educación y por lo tanto sus dispositivos de control. Hoy asistimos entonces al surgimiento de nuevos discursos, cuyo carácter dominante es la evaluación, en torno al cual se definen los otros asuntos de la enseñanza y el aprendizaje.

Estos escenarios están produciendo discursos, a través de los cuales ponen a disposición, propósitos, teorías, conceptos, metodologías y recursos para la enseñanza de la historia desde su propia historia. El lugar que ocupen, la manera como se organicen, las relaciones que se establezcan, la visibilidad o el ocultamiento que obtengan, serían los rasgos que darían cuenta de la manera como se configura ese saber y del tipo de saber pedagógico de los maestros para los propósitos que nos convocan.

Tradicionalmente, los estudios sobre la Enseñanza de la Historia en Colombia se han regido por tres rutas discursivas distintas; el análisis de las políticas educativas relacionadas con el campo², los balances acerca de la producción académica ${ }^{3}$, y a través de los análisis de los manuales escolares ${ }^{4}$. Si bien, han sido estudios que develan importantes desarrollos y movimientos sobre la manera cómo ha tomado forma la enseñanza de la historia en el país. La indagación de estas perspectivas difícilmente da cuenta de la compleja red de conceptos, sentidos, propósitos, teorías, didácticas, saberes, contextos, entre muchos otros elementos, que un docente debe conocer, unir, relacionar y organizar para disponerlo de alguna manera, para su enseñanza. En pequeños ejercicios que hemos realizado con los 
estudiantes de la Carrera de Historia de la Pontificia Universidad Javeriana, hemos venido constatando que tal complejidad no sólo está asociada a la amplia dispersión de su discurso, sino fundamentalmente, a las tensiones internas que lo atraviesan, tanto en sus posturas teóricas y sus opciones metodológicas, como por sus demandas políticas y sociales.

Esta exigencia podría ser constitutiva al oficio de maestro en la actualidad, en tanto la "crisis" del proyecto moderno que políticamente giraba en torno a esa "comunidad imaginada" llamada "nación", ha interpelado críticamente lo que le era sustancial, tanto la escuela como el maestro. Podríamos arriesgarnos a asegurar, que para aquellos sujetos que soportan el saber pedagógico de la enseñanza de la historia, o de las ciencias sociales, tal exigencia se ha incrementado por cuanto esa crisis también ha interpelado su saber, en particular en sus propósitos éticos y políticos en la escuela. Si entendemos, como lo plantea Zermeño (2002: 16), que la historia es la "autobiografía de la modernidad", podemos dimensionar lo que significa la crisis de la historia y de las ciencias sociales como saber escolar en el proyecto actual.

Estamos pensando en el saber como "[...] el conjunto de conocimientos de niveles desiguales (cotidianos o con pretensiones de teóricos), cuyos objetos son enunciados en diversas prácticas y por sujetos diferentes [...]" (Zuluaga, 1999: 148). Continuando con Zuluaga podemos afirmar que el saber adjetivado como pedagógico lo definimos como "[...] el conjunto de conocimientos con estatuto teórico o práctico, que conforman un domino de saber institucionalizado el cual configura la práctica de la enseñanza y la adecuación de la educación en la sociedad [...]" (1999: 148-149). Por lo tanto, metodológicamente se deberá proceder a identificar los discursos, sujetos y prácticas que atraviesan el saber pedagógico sobre la enseñanza de la historia en la actualidad, para tratar de develar las diferentes narrativas que hacen parte del campo discursivo de la enseñanza de la historia en la escuela. Tal vez, la novedad sería plantear las posibles relaciones y vínculos entre estas narrativas para avanzar en la comprensión sobre la manera cómo se organizan como saber, y por último, intentar describir las formas que toma este saber pedagógico hoy.

Por lo tanto, para visibilizar el estado de la práctica pedagógica en la enseñanza de la historia en la Educación Básica, sería necesario, avanzar en el establecimiento de posibles diálogos entre los procesos y resultados de los estudios realizados, con otro campo que también incide en la configuración de la práctica pedagógica, como es la formación de docentes. Pero también, faltaría relacionar esta producción con un trabajo etnográfico con docentes de diferentes 
niveles, formaciones y trayectorias, que avance en la identificación de configuraciones diferentes, que nos proponga otros diálogos y debates. Este trabajo estaría por hacer.

Las anteriores urgencias se proponen a la luz de hacer más franqueables las rupturas, las fragmentaciones y los distanciamientos entre lo que sucede en el aula escolar y la manera como desde otros escenarios (de política, académicos, de formación, de evaluación, etc.) se comprende y se prescribe lo que hace el maestro en ella. Abordar cada uno de los campos antes relacionados, seguramente complejizará la mirada a través de la cual podamos construir otros abordajes sobre el sentido de ser maestro hoy, que en lo fundamental, nos ayude a intentar superar las valoraciones sociales relacionadas con la inamovilidad de los docentes, el poco compromiso y su "resistencia" al cambio.

Así como ninguno de los abordajes particulares de cada campo, podría recoger la complejidad de lo que ha significado la enseñanza, ante la envergadura de lo propuesto, por ahora, solo intentaremos esbozar algunos elementos relacionados con la manera cómo se ha configurado históricamente el "saber pedagógico" de los maestros para la enseñanza de la historia: ¿Qué constituye el saber pedagógico de un maestro hoy para la enseñanza de la historia? ¿Cómo se ha configurado? y ¿Qué formas toma ese saber pedagógico hoy para la enseñanza de la historia?

Adoptando la periodicidad propuesta por Álvarez (2007) y Martínez (2007) identificamos tres momentos de la Enseñanza de la historia. El primero, entre la patria y la nación (1870- 1960), en el que la institucionalización de los estados nacionales fue un acontecimiento íntimamente ligado a la escolarización de la población y en particular, a la centralidad de la enseñanza de la historia en la escuela. La educación, la escuela y por supuesto la historia como saber escolar, estuvieron al servicio de la nación: ayudaron a inventarla y se constituyeron en el camino hacia la civilización (Álvarez, 2007).

Un segundo momento, en el que la enseñanza de la historia transita por el "reino" de la ciencia y del desarrollo (1960-1990), en el que el Estado cambia su lugar y su estrategia de control sobre la enseñanza de la historia. El control estatal dirigió y decidió sobre los contenidos y metodologías, a través de la formulación de planes de estudio o marcos generales, pretendiendo cada vez más, tener un control sobre el quehacer del docente; compartió la inspección sobre los textos escolares con la Academia Colombiana de Historia y se desentendió, casi completamente, de la formación inicial de docentes, pues ésta quedó en manos de las nacientes facultades de educación. 
Al terminar el periodo, es decir, hacia finales de los años ochenta, encontramos una gran preocupación sobre la formación de los docentes en ejercicio, así como un amplio debate sobre la elaboración de los textos escolares.

Y un tercer momento, donde la enseñanza de la historia será parte de la crisis de los discursos sobre el saber, los sujetos y las instituciones, periodo que comprende desde 1990 hasta hoy, donde podríamos preguntarnos ¿Cuál sería el aporte de un saber escolar, como la historia, para la formación de individuos cuyo propósito ya no es el fortalecimiento de la identidad y el compromiso con un proyecto nacional, tampoco con la democracia y la ciudadanía, y ahora, se le requiere como un agente para la productividad y la competitividad?

De manera general, vemos que el sentido de la enseñanza de la historia hasta hoy, se ha movido por dos cauces: el primero, su función moral, sentimental y afectiva asociada con la manera cómo se narran los sujetos, sea en clave de patria, nación, estado, democracia o ciudadanía; cuyo propósito ha sido el de crear vínculos, pertenencia, identidad o identidades; el segundo se relaciona con su función científica, racional y de formación de pensamiento, relacionada con los "mandatos" que los Estados y las sociedades han construido, en torno a sus ideas de civilización, progreso, desarrollo, competitividad o productividad, donde la escuela, el maestro y la historia, son el dispositivo ya sea para la adquisición de conocimientos sobre la nación, o de habilidades para el desarrollo o de competencias para el mercado.

Un tercer cauce toma forma desde los años sesentas, relacionado con las posibilidades transformadoras de las sociedad, donde la historia permite su comprensión "profunda"; este camino toma elementos de los dos anteriores. Del primero su posibilidad de movilizar sentimientos y valores a través de otras narraciones sobre otros sujetos, esta vez, en clave de justicia, igualdad y solidaridad, donde no necesariamente el referente es la nación o la ciudadanía. Del segundo recoge su convicción por el rigor, la sistematicidad, el manejo de fuentes y las perspectivas críticas de la historia, orientada a fines propositivos de denuncia o de militancia.

En relación con el contenido de la historia que se enseña, difícilmente se ha podido desmarcar de las convenciones que le señalan lo "científico" del conocimiento, que para la escuela en particular, están ancladas en la verdad, la objetividad, la neutralidad y el método. Por esta vía, discurren los diferentes desplazamientos en los contenidos de la enseñanza de la historia: han transitado de unos héroes, acontecimientos y fechas a unos procesos históricos; de unos contenidos de historia a unos historiográficos; de unos contenidos 
conceptuales a otros procedimentales y de orden valorativo, y por último de unas temáticas históricas a unas problemáticas sociales.

Y con respecto al cómo enseñar, es indudable la contundencia de la huella pestalozziana, afiliada a la herencia de la pedagogía racional sobre "lo natural en la forma de conocer". A pesar de la sofisticación que en algunos momentos alcanzaron los diseños temáticos y luego los curriculares ya fueran instruccionales por objetivos, por indicadores de logros, por competencias y en este siglo XXI, por estándares; la "prisión pedagógica" sigue vigente, actualizándose en la pedagogía activa y en el constructivismo. La única fractura, pero con poca consistencia práctica, ha sido la propuesta de Lineamientos Curriculares del año 2002.

Pero la configuración del saber de la historia para su enseñanza, se juega no solamente en su propio terreno, sino también en el de las ciencias sociales donde se cuestiona la visión disciplinar, especialmente - añadiríamos-, para su enseñanza. Sin embargo, hemos insistido en ella, para hacer visible la fuerza de esta discursividad anclada en torno a la nación, la escuela y la historia, pues se ha privilegiado la función social de su discurso antes que la científica. No obstante, desde el año 2004, la formulación de los Estándares Básicos de Competencias en ciencias sociales, hace notable la convergencia de estas dos discursividades cuando se plantea que "[...] formar en ciencias significa contribuir a la formación de ciudadanos y ciudadanas capaces de razonar, debatir, producir, convivir y desarrollar al máximo su potencial creativo [...]" (Ministerio de Educación Nacional, 2004: 6).

No obstante, la forma que toma el saber pedagógico para la enseñanza de la historia o de las ciencias sociales hoy, se amplía y complejiza significativamente al considerar otras áreas de lo social que se han formalizado para el currículo escolar: la cátedra de estudios afrocolombianos, la constitución política, la educación ambiental y las competencias ciudadanas, la memoria histórica, por mencionar algunas. Este saber pedagógico, de por sí ya complejo, se reconfigura en la escuela, en el territorio, por cuanto allí lo social tiene otras connotaciones, y en consecuencia, también la enseñanza de la historia y de las ciencias sociales. Entendiendo a éstas como instrumentos fundamentales para el conocimiento de la sociedad, el área y los sujetos que la soportan, sitúan su saber tratando de responder a otras expectativas escolares que tiene que ver con otros asuntos: los problemas de convivencia, la paliación de traumas (familiares, comunitarios), la gobernabilidad en la escuela, entre muchos otros.

La pregunta, entonces, sobre lo que se le pide al maestro hoy -la cual podría formularse interrogando ¿qué tipo de maestro se nece- 
sita para la enseñanza de la historia y de las ciencias sociales?-, implica reconocer que los maestros requieren del dominio de más saberes de los demandados en otro tiempo en la escuela. El saber pedagógico toma forma a través de los sujetos de la enseñanza, y a la vez ellos se han configurado en su relación con el saber de diversas formas: como habilidosos artesanos cuidadores de su oficio que pacientemente tejían los relatos sobre la historia; expertos planeadores que dosifican y proponían un ordenamiento "lógico" a los contenidos de la historia; trabajadores culturales incansables que se ocupan de mediar entre la historia "científica" y la historia "popular"; facilitadores que acondiciona los ejes curriculares o las preguntas poblematizadoras para administrar un currículo; el investigador que hace de la ciencia social su práctica de enseñanza; el intelectual que hace consciencia de su condición histórica y la vuelve el eje de su crecimiento, o el profesional al que se le pide ser competente en los asuntos a su cargo. Todos esos posicionamientos del sujeto maestro hacen síntesis en él, pero también se juegan estratégicamente en los diferentes espacios por los que transita. Aunque también la política educativa, los investigadores universitarios, los medios de comunicación, los empresarios y las universidades, hace buen uso de ellos.

El saber pedagógico para la enseñanza de la historia y de las ciencias sociales, toma forma en la manera como se organizan institucionalmente los saberes y los sujetos, de una parte a través de las Facultades de Educación y las Escuelas Normales Superiores que hablan de una especialización de la enseñanza, o de otros escenarios, como los departamentos de historia en los que se habla de la especialización disciplinar. Ninguna de las dos agota la complejidad del saber pedagógico para la enseñanza de la historia y de las ciencias sociales. Será desde la escuela, en su experiencia, que los maestros construyen otros saberes relacionados por ejemplo, a un saber institucional asociado con el ejercicio de leer el interior de la escuela, descifrar sus lógicas y los sentidos presentes en ella y definir el qué y cómo, insertar los saberes "propios del área".

Fecha de recepción: 15 de octubre de 2011

Fecha de evaluación: 24 de septiembre de 2012

Fecha de aprobación: 18 de octubre de 2012

Cómo citar este artículo: González, M. (2012). La historia de la enseñanza de la historia: un saber escolar inestable. Praxis Pedagógica 13: 56-73. 
㗊 Referencias

Álvarez, A. (2003). Los medios de comunicación y la sociedad educadora ¿Ya no es necesaria la Escuela?. Bogotá: Editorial Magisterio y Universidad Pedagógica Nacional.

- Álvarez, A. (2007). Las ciencias sociales en el currículo escolar. 19301960. Tesis de Doctorado en Historia de la Educación y Educación Comparada, Universidad Nacional a Distancia.

- Álvarez, A. y León Palencia, A. C. (2008). ¿Para qué formar maestros? En González Lara, M. ( Ed.). Paradojas en la Formación Docente. Bogotá: Editorial Santa María, Organización de Estados Iberoamericanos (OEI), Gobierno de Aragón.

- Betancourt, A. (2007). Historia y nación, tentativas de la escritura de la historia en Colombia. Medellín: La Carreta.

- Castillo, E. (2010). Conmemorando otro Bicentenario. Periódico de Pensamiento Universitario para la Sociedad., Universidad del Cauca, 22.

- Certeau, M. de. (2006). La escritura de la historia. México: Universidad Iberoamericana.

- Cortés, R. A., González Lara, M. y Ospina, C. (2001). La enseñanza de la historia, ¿una cuestión de la historia, o una historia cuestionada? Balance de una década. Bogotá: Ponencia X Congreso Colombiano de Historia.

Cuesta Fernández, R. (1997). Sociogénesis de una disciplina escolar: La Historia. Barcelona: Pomares-Corredor.

- CEPECS. (1985). Ciencias sociales y proyecto pedagógico alternativo. IV Seminario Nacional de Educción y Sociedad, CEPECS.

- Gualteros M. I. (2005). El texto escolar Historia de Colombia de Henao y Arrubla y su relación con el método pedagógico utilizado en Colombia entre 1800-1910. Trabajo de Grado, Carrera de Historia, Universidad Javeriana.

- Lander, E. (s.f.). Ciencias Sociales: saberes coloniales y eurocéntricos. Caracas: Universidad Central de Venezuela.

- Lombana, H. A. (2007). Ciencias Sociales y Reforma curricular: análisis comparativo de los diseños curriculares de 1984 y 2002. Tesis de Grado, Maestría en Sociología de la Educación, Universidad Distrital Francis José de Caldas. 
- Martínez, A. (2004). De la escuela expansiva a la escuela competitiva: modos de modernización en América latina. Bogotá: Convenio Andrés Bello.

Ministerio de Educación Nacional, OEI. (2002). Lineamientos Curriculares en Ciencias Sociales. Bogotá: Ministerio de Educación Nacional.

- Ministerio de Educación Nacional. (2004). Formar en Ciencias; ¡el desafío! Lo que necesitamos saber y saber hacer. Serie Guía No. 7. Estándares Básicos de Competencias en Ciencias Naturales y Ciencias Sociales. Bogotá: Ministerio de Educación Nacional.

- $\quad$ Ospina de F. C. (2007). Análisis del texto de Vergara y Velasco, Francisco Javier. Novísimo texto de Historia de Colombia (Historia Patria). Segunda edición completamente refundida, Editorial, Librería Colom biana y Librería Nueva, Bogotá, 1910. Documento Versión Digital.

· $\quad$ Ossenbach G. y Somoza M. (eds.). (2001). Los Manuales escolares como fuente para la historia de la educación en América Latina. Madrid: Universidad Nacional de Educación a Distancia.

- Rodríguez, S. P. y Acosta, W. (2008). Autores, problemas y debates en la investigación sobre la enseñanza de la historia: 1990-2006. Ponencia X Jornada Nacional y I Encuentro internacional sobre la Enseñanza de la Historia, Universidad Nacional de Rio Cuarto.

- Saldarriaga, O. (2003). Del oficio de maestro. Prácticas y teoría de la pedagogía moderna en Colombia. Bogotá: Editorial Magisterio.

- Tovar González, L. (2002). Ciencia y fe: Miguel Antonio Caro y las ideas positivistas. Bogotá: Universidad Nacional.

- Zapata, V. (2003). La evolución del concepto de saber pedagógico: su ruta de transformación. Revista Educación y Pedagogía, XV (37).

- Zermeño, G. (2002). La cultura moderna de la historia. Una aproximación teórica e historiográfica. México: El Colegio de México.

· Zuluaga, O. L. (1999). Pedagogía e historia. Medellín: Editorial Universidad de Antioquia, Anthropos, Siglo del Hombre Editores. 